\title{
DECOMPOSITIONS OF MEASURES ON PSEUDO EFFECT ALGEBRAS
}

\author{
ANATOLIJ DVUREČENSKIJ \\ 1 Mathematical Institute, Slovak Academy of Sciences \\ Štefánikova 49, SK-814 73 Bratislava, Slovakia \\ E-mail: dvurecen@mat.savba.sk,
}

\begin{abstract}
Recently in Dvu3 it was shown that if a pseudo effect algebra satisfies a kind of the Riesz Decomposition Property ((RDP) for short), then its state space is either empty or a nonempty simplex. This will allow us to prove a Yosida-Hewitt type and a Lebesgue type decomposition for measures on pseudo effect algebra with (RDP). The simplex structure of the state space will entail not only the existence of such a decomposition but also its uniqueness.
\end{abstract}

\section{INTRODUCTION}

The classical decomposition theorems of or Yosida-Hewitt $\mathrm{YoHe}$ initiated in the last two decades interest of authors studying finitely additive measures on quantum structures like orthomodular lattices or posets to study an interesting problem of decomposition measures. There appeared a whole series of papers studying Lebesgue and Yosida-Hewitt type decompositions, see e.g. DDP, DeMo, Rut1, Rut2. They exhibited at least the existence of such a decomposition. To prove even the uniqueness of decompositions, some sufficient conditions are presented in Rut2.

Quantum structures were inspired by the research of the mathematical foundations of quantum structures. An analogue of a probability measure is a state. One of the most important examples of orthomodular lattices or of the Hilbert space quantum mechanics is the system $\mathcal{L}(H)$ of all closed subspaces of a Hilbert space (real, complex or quaternionic) $H$. The Gleason Theorem, see e.g. Dvu0, says that every $\sigma$-additive state on $\mathcal{L}(H)$ is uniquely expressible via a Hermitian trace operator on $H$ if $3 \leq \operatorname{dim} H \leq \aleph_{0}$. The Aarnes Theorem, [Dvu0, Thm 3.2.28] says that every (finitely additive) state on $\mathcal{L}(H)$ is a unique convex combination of two states, $s_{1}$ and $s_{2}$, where $s_{1}$ is a completely additive state and $s_{2}$ is a finitely additive state that vanishes on each finite-dimensional subspace of $H$.

In the Nineties, Foulis and Bennett [FoBe] introduced effect algebras that are partial structures with a partially defined operation + that models the join of mutually excluding events. They generalize orthomodular lattices and posets, orthoalgebras,

\footnotetext{
${ }^{1}$ Keywords: Pseudo effect algebra; effect algebra; Riesz Decomposition Properties; signed measure, state; Jordan signed measure, unital po-group; simplex; Yosida-Hewitt decomposition; Lebesgue decomposition

AMS classification: 81P15, 03G12, 03B50

The author thanks for the support by Center of Excellence SAS - Quantum Technologies -, ERDF OP R\&D Projects CE QUTE ITMS 26240120009 and meta-QUTE ITMS 26240120022, the grant VEGA No. 2/0032/09 SAV.
} 
and the basic example important for so-called POV-measures of quantum mechanics is the system, $\mathcal{E}(H)$, of all Hermitian operators of a Hilbert space $H$ that are between the zero operator and the identity one.

These commutative structures were extended in DvVe1, DvVe2] to so-called pseudo effect algebras where the partial addition, + , is not more assumed to be commutative. In many important examples they are intervals in po-groups $(=$ partially ordered groups). E.g. $\mathcal{E}(H)$ is an interval in the po-group $\mathcal{B}(H)$ of all Hermitian operators on $H$.

A sufficient condition for a pseudo effect algebra to be an interval of a unital pogroup is a variant of the Riesz Decomposition Property ((RDP) in abbreviation), see Dvu2, DvVe2. It is a weaker form of the distributivity that allows to do a joint refinement of two decompositions of the unit element 1. For example, (RDP) on an orthomodular poset entails that it has to be a Boolean algebra, and therefore, (RDP) fails to hold on $\mathcal{L}(H)$ or on $\mathcal{E}(H)$.

We recall that every effect algebra with (RDP) has at least one state, however the state space of a pseudo effect algebra with a stronger type of (RDP) can be empty, Dvu1.

Recently in Dvu3, Thm 5.1], it was shown that the state space of every pseudo effect algebra with (RDP) is a simplex, more precisely a Choquet simplex. The simplex is a special type of a convex set that generalizes the classical one in $\mathbb{R}^{n}$. For a comprehensive source on simplices see [Alf. We note that the state space of $\mathcal{E}(H)$ is not a simplex, however the state space of any commutative $\mathrm{C}^{*}$-algebra is a simplex, see e.g. [AlSc, Thm 4.4, p. 7]. The simplex structure of the state spaces allows also to represent uniquely states as an integral Dvu4 through a regular Borel probability measure.

The simplex structure of the state space of a pseudo effect algebra $E$ satisfying (RDP) entails that the space of all Jordan measures on $E$ is an Abelian Dedekind complete $\ell$-group, Dvu3, Thm 3.5, Thm 3.6]. This new fact is our basic tool to present a Yosida-Hewitt type and a Lebesgue type of decompositions of finitely additive measures on $E$. The property (RDP) as we show is a sufficient condition to prove not only the existence but also the uniqueness of such a decomposition that is a main goal of the present paper.

The paper is organized as follows.

Section 2 is an introduction to the theory of pseudo effect algebras gathering the necessary latest results. Section 3 describes the faces of the state space of pseudo effect algebras and it gives a general result on a decomposition and it will be applied in Section 4 to present the main body of the paper - the Yosida-Hewitt type of decomposition and the Lebesque type decomposition.

\section{Elements of Pseudo Effect Algebras}

Pseudo effect algebras were introduced in DvVe1, DvVe2. We say that a pseudo effect algebra is a partial algebra $(E ;+, 0,1)$, where + is a partial binary operation and 0 and 1 are constants, such that for all $a, b, c \in E$, the following holds

(i) $a+b$ and $(a+b)+c$ exist if and only if $b+c$ and $a+(b+c)$ exist, and in this case $(a+b)+c=a+(b+c)$;

(ii) there is exactly one $d \in E$ and exactly one $e \in E$ such that $a+d=e+a=1$;

(iii) if $a+b$ exists, there are elements $d, e \in E$ such that $a+b=d+a=b+e$;

(iv) if $1+a$ or $a+1$ exists, then $a=0$. 
If we define $a \leq b$ if and only if there exists an element $c \in E$ such that $a+c=b$, then $\leq$ is a partial ordering on $E$ such that $0 \leq a \leq 1$ for any $a \in E$. It is possible to show that $a \leq b$ if and only if $b=a+c=d+a$ for some $c, d \in E$. We write $c=a / b$ and $d=b \backslash a$. Then

$$
(b \backslash a)+a=a+(a / b)=b,
$$

and we write $a^{-}=1 \backslash a$ and $a^{\sim}=a / 1$ for any $a \in E$.

For basic properties of pseudo effect algebras see DvVe1, DvVe2. We recall that if + is commutative, $E$ is said to be an effect algebra; for a guide overview on effect algebras we recommend e.g. [DvPu.

For example, let $(G, u)$ be a unital po-group (= partially ordered group) with strong unit $u$ that is not necessarily Abelian. We recall that a po-group (= partially ordered group) is a group with a partial ordering $\leq$ such that if $a \leq b$, then $x+a+$ $y \leq x+b+y$ for all $x, y \in G$; and an element $u \in G^{+}:=\{g \in G: g \geq 0\}$ is said to be a strong unit if given $G=\bigcup_{n}[-n u, n u]$.

Then for $(G, u)$ we define $\Gamma(G, u)=[0, u]$ and we endow it with + that is the restriction of the group addition to the set of all those $(x, y) \in \Gamma(G, u) \times \Gamma(G, u)$ that $x \leq u-y$. Then $(\Gamma(G, u) ;+, 0, u)$ is a pseudo effect algebra with possible two negations: $a^{-}=u-a$ and $a^{\sim}=-a+u$. Any pseudo effect algebra of the form $\Gamma(G, u)$ is said to be an interval pseudo effect algebra. In DvVe1, DvVe2], we have some sufficient conditions posed to a pseudo effect algebra to be an interval. They are analogues of the Riesz Decomposition Properties. Roughly speaking they are a weaker form of distributivity that allows a joint refinement of two partitions of 1 . This is a reason why they fail to hold for $\mathcal{L}(H)$ and $\mathcal{E}(H)$.

Now we introduce according to DvVe1 the following types of the Riesz Decomposition properties for pseudo effect algebras that in the case of effect algebras may coincide, but not for pseudo effect algebras, in general.

(a) For $a, b \in E$, we write $a \operatorname{com} b$ to mean that for all $a_{1} \leq a$ and $b_{1} \leq b, a_{1}$ and $b_{1}$ commute.

(b) We say that $E$ fulfils the Riesz Interpolation Property, (RIP) for short, if for any $a_{1}, a_{2}, b_{1}, b_{2} \in E$ such that $a_{1}, a_{2} \leq b_{1}, b_{2}$ there is a $c \in E$ such that $a_{1}, a_{2} \leq c \leq b_{1}, b_{2}$.

(c) We say that $E$ fulfils the weak Riesz Decomposition Property, $\left(\mathrm{RDP}_{0}\right)$ for short, if for any $a, b_{1}, b_{2} \in E$ such that $a \leq b_{1}+b_{2}$ there are $d_{1}, d_{2} \in E$ such that $d_{1} \leq b_{1}, d_{2} \leq b_{2}$ and $a=d_{1}+d_{2}$.

(d) We say that $E$ fulfils the Riesz Decomposition Property, (RDP) for short, if for any $a_{1}, a_{2}, b_{1}, b_{2} \in E$ such that $a_{1}+a_{2}=b_{1}+b_{2}$ there are $d_{1}, d_{2}, d_{3}, d_{4} \in$ $E$ such that $d_{1}+d_{2}=a_{1}, d_{3}+d_{4}=a_{2}, d_{1}+d_{3}=b_{1}, d_{2}+d_{4}=b_{2}$.

(e) We say that $E$ fulfils the commutational Riesz Decomposition Property, $\left(\mathrm{RDP}_{1}\right)$ for short, if for any $a_{1}, a_{2}, b_{1}, b_{2} \in E$ such that $a_{1}+a_{2}=b_{1}+b_{2}$ there are $d_{1}, d_{2}, d_{3}, d_{4} \in E$ such that (i) $d_{1}+d_{2}=a_{1}, d_{3}+d_{4}=a_{2}$, $d_{1}+d_{3}=b_{1}, d_{2}+d_{4}=b_{2}$, and (ii) $d_{2} \operatorname{com} d_{3}$.

(f) We say that $E$ fulfils the strong Riesz Decomposition Property, $\left(\mathrm{RDP}_{2}\right)$ for short, if for any $a_{1}, a_{2}, b_{1}, b_{2} \in E$ such that $a_{1}+a_{2}=b_{1}+b_{2}$ there are $d_{1}, d_{2}, d_{3}, d_{4} \in E$ such that (i) $d_{1}+d_{2}=a_{1}, d_{3}+d_{4}=a_{2}, d_{1}+d_{3}=b_{1}$, $d_{2}+d_{4}=b_{2}$, and (ii) $d_{2} \wedge d_{3}=0$.

We have the implications

$$
\left(\mathrm{RDP}_{2}\right) \Rightarrow\left(\mathrm{RDP}_{1}\right) \Rightarrow(\mathrm{RDP}) \Rightarrow\left(\mathrm{RDP}_{0}\right) \Rightarrow(\mathrm{RIP})
$$


The converse of any of these implications does not hold. For commutative effect algebras we have

$$
\left(\mathrm{RDP}_{2}\right) \Rightarrow\left(\mathrm{RDP}_{1}\right) \Leftrightarrow(\mathrm{RDP}) \Leftrightarrow\left(\mathrm{RDP}_{0}\right) \Rightarrow(\mathrm{RIP}) .
$$

If in the above definitions of (RDP)'s we change $E$ to $G^{+}$, we have po-groups with the corresponding forms of the Riesz Decomposition Properties. According to DvVe2, Thm 5.7], every pseudo effect algebra with (RDP) $)_{1}$ is an interval in some unital po-group with $(\mathrm{RDP})_{1}$, so is any effect algebra with (RDP) in an Abelian po-group with (RDP), see see $[\mathrm{Rav}]$ or [DvPu, Thm 1.7.17]. Any effect algebra with (RDP) that is a lattice, or equivalently, with $(\mathrm{RDP})_{2}$ is an MV-algebra, for a definition see e.g. $\mathrm{DvPu}$. Any pseudo effect algebra with $(\mathrm{RDP})_{2}$ is a so-called pseudo MV-algebra, see GeIo, and the group $G$ is an $\ell$-group (= lattice ordered group).

A signed measure on a pseudo effect algebra $E$ is any mapping $m: E \rightarrow \mathbb{R}$ such that $m(a+b)=m(a)+m(b)$ provided $a+b$ is defined in $E$. We have $s(0)=0$ and $s\left(a^{-}\right)=s\left(a^{\sim}\right)$. If a signed measure $m$ is positive, i.e., $m(a) \geq 0$ for each $a \in E$, we call it a measure, and any normalized measure, i.e. a measure $s$ such that $s(1)=1$, is said to be a state. For any measure $m$, we have $m(a) \leq m(b)$ whenever $a \leq b$. If $m_{1}$ and $m_{2}$ are two measures on $E$, then the signed measure $m=m_{1}-m_{2}$ is said to be a Jordan signed measure. We denote by $\mathcal{M}(E), \mathcal{M}^{+}(E), \mathcal{S}(E)$, and $\mathcal{J}(E)$ the sets of all signed measures, or measures, or states or Jordan signed measures on $E$. We recall that it can happen that $\mathcal{M}(E)=\{0\}=\mathcal{J}(E)$.

The set $\mathcal{S}(E)$ is convex, i.e., any convex combination $s=\lambda s_{1}+(1-\lambda) s_{2}, \lambda \in[0,1]$, of two states $s_{1}$ and $s_{2}$ and $\lambda \in[0,1]$ is a state. If $s$ cannot be expressed by a convex combination of two distinct states, it is called an extremal state. Let $\partial_{e} \mathcal{S}(E)$ denote the set of all extremal states. On $\mathcal{M}(E)$ we introduce a weak topology: We say that a net of measures $\left\{m_{\alpha}\right\}$ converges weakly to a measure $m$ if $\lim _{\alpha} m_{\alpha}(a)=m(a)$. Then $\mathcal{S}(E)$ is a convex compact Hausdorff space, and due to the Krein-Mil'man Theorem, see [Goo, Thm 5.17], every state on $E$ is a weak limit of a net of convex combinations of extremal states.

If $E$ is a pseudo effect algebra with (RDP), then $\mathcal{S}(E)$ is either empty or a nonvoid simplex, Dvu3, Thm 5.1], for definition of a simplex and its basic properties, see Goo, Chap 10].

For two signed measures $m_{1}$ and $m_{2}$, we write $m_{1} \leq{ }^{+} m_{2}$ if $m_{1}(a) \leq m_{2}(a)$ for each $a \in E$.

The following important statement was proved in [Dvu3, Thm 3.5, Thm 3.6]:

Theorem 2.1. Let $E$ be a pseudo effect algebra with (RDP). Then $\mathcal{J}(E)$ is an Abelian Dedekind complete $\ell$-group such that if $\left\{m_{i}\right\}_{i \in I}$ is a nonempty system of $\mathcal{J}(E)$ that is bounded above, and if $d(x)=\bigvee_{i} m_{i}(x)$ for all $x \in E$, then

$$
\left(\bigvee_{i} m_{i}\right)(x)=\bigvee\left\{d\left(x_{1}\right)+\cdots+d\left(x_{n}\right): x=x_{1}+\cdots+x_{n}, x_{1}, \ldots, x_{n} \in E\right\}
$$

for all $x \in E$.

And if $e(x)=\bigwedge_{i} f_{i}(x)$ for all $x \in E$, then

$$
\left(\bigwedge_{i} m_{i}\right)(x)=\bigwedge\left\{e\left(x_{1}\right)+\cdots+e\left(x_{n}\right): x=x_{1}+\cdots+x_{n}, x_{1}, \ldots, x_{n} \in E\right\}
$$

for all $x \in E$.

Given $m_{1}, \ldots, m_{n} \in \mathcal{J}(E)$, 


$$
\begin{aligned}
& \left(\bigvee_{i=1}^{n} m_{i}\right)(x)=\sup \left\{m_{1}\left(x_{1}\right)+\cdots+m_{n}\left(x_{n}\right): x=x_{1}+\cdots+x_{n}, x_{1}, \ldots, x_{n} \in E\right\}, \\
& \left(\bigwedge_{i=1}^{n} m_{i}\right)(x)=\inf \left\{m_{1}\left(x_{1}\right)+\cdots+m_{n}\left(x_{n}\right): x=x_{1}+\cdots+x_{n}, x_{1}, \ldots, x_{n} \in E\right\},
\end{aligned}
$$

for all $x \in E$.

\section{Faces of the State Space}

The present section describes the faces of the state space of a pseudo effect algebra with (RDP). Since our state space is a simplex, we know that if $F$ is a closed face, then every simplex is a direct convex sum of $F$ and its complementary face, Goo, Thm 11.28]. However, not every face is closed, we present a general decomposition, Theorem 3.4 where a weaker form of the closedness, $\vee$-closedness, allows to obtain a unique decomposition of measures. This result will apply in the next section to obtain Yosida-Hewitt and Lebesgue types of decomposition.

A face of a convex set $K$ is a convex subset $F$ of $K$ such that if $x=\lambda x_{1}+(1-$ $\lambda) x_{2} \in F$ for $\lambda \in(0,1)$, then also $x_{1}, x_{2} \in F$. We note that if $x \in K$, then $\{x\}$ is a face iff $x \in \partial_{e} K$.

For any $X \subseteq K$, there is the face generated by $X$. Due to [Goo, Prop 5.7], the face $F$ generated by $X$ is the set of those points $x \in K$ for which there exists a positive convex combination $\lambda x+(1-\lambda) y=z$ with $y \in K$ and $z$ belongs to the convex hull of $X$.

If $\mathcal{S}(E) \neq \emptyset$, then a state $s \in \mathcal{S}(E)$ belongs to the face generated by $X$ if and only if $s \leq^{+} \alpha t$ for some positive constant $\alpha$ and some state $t$ in the convex hull of $X$. In particular, the face of $\mathcal{S}(E)$ generated by a state $s$ is the set of states $s^{\prime} \in \mathcal{S}(E)$ such that $s^{\prime} \leq^{+} \alpha s$ for some real number $\alpha>0$.

Let $s$ be a state on a pseudo effect algebra $E$. The kernel of $s$ is the set

$$
\operatorname{Ker}(s):=\{x \in E: s(x)=0\} .
$$

Then $\operatorname{Ker}(s)$ is a normal ideal of $E$. We note that a subset $I$ of a pseudo effect algebra is an ideal if (i) $0 \in I$, (ii) $a, b \in I$ and $a+b \in E$ imply $a+b \in I$, and (iii) $a \in E, b \in I$ and $a \leq b$ entail $a \in I$. An ideal $I$ is normal, if $a+I:=\{a+b \in E$ : $b \in I\}=I+a:=\{b+a \in E: b \in I\}$ for any $a \in E$.

Proposition 3.1. Let $E$ be a pseudo effect algebra and let $X$ be a subset of $E$. Then the set

$$
F=\{s \in \mathcal{S}(E): X \subseteq \operatorname{Ker}(s)\}
$$

is a closed face of $\mathcal{S}(E)$.

Proof. If $F=\emptyset$, then $F$ is trivially a closed face. Assume $F \neq \emptyset$. If $s=\lambda s_{1}+(1-$ $\lambda) s_{2}$ for $\lambda \in[0,1]$ and for two states $s_{1}$ and $s_{2}$, then $\operatorname{Ker}(s) \subseteq \operatorname{Ker}\left(s_{1}\right) \cap \operatorname{Ker}\left(s_{2}\right)$ which proves that $F$ is a convex set. If $\left\{s_{\alpha}\right\}$ is a net of states from $F$ that converges weakly to a state $s$ on $E$, then for each $x \in X, 0=\lim _{\alpha} s_{\alpha}(x)=s(x)$ so that $F$ is closed. If now $s=\lambda s_{1}+(1-\lambda) s_{2} \in F$ for $\lambda \in(0,1)$ and $s_{1}, s_{2} \in F$, then for each $x \in X$ we have $0=s(x)=\lambda s_{1}(x)+(1-\lambda) s_{2}(x)$ so that $s_{1}(x)=s_{2}(x)=0$ and therefore, $s_{1}, s_{2} \in F$. 
Let $F$ be a face of a simplex $K$, and let $F^{\prime}$ be the union of those faces of $K$ that are disjoint from $F$. According to Goo, Prop 10.12], $F^{\prime}$ is a face of $K$ and it is the largest face of $K$ that is disjoint from $F$. If a point $x \in K$ can be expressed as convex combinations

$$
x=\alpha_{1} x_{1}+\alpha_{2} x_{2}=\beta_{1} y_{1}+\beta_{2} y_{2}
$$

with $x_{1}, y_{1} \in F$ and $x_{2}, y_{2} \in F^{\prime}$, then $\alpha_{i}=\beta_{i}$ for $i=1,2$ and $x_{i}=y_{i}$ for those $i$ such that $\alpha_{i}>0$. The face $F^{\prime}$ is said to be a complementary face of $F$ in $K$.

It is possible to show that if $F_{1} \subseteq F_{2}$ are two faces of $\mathcal{S}(E)$, then $F_{2}^{\prime} \subseteq F_{1}^{\prime}$ and for a set $\left\{F_{i}\right\}$ of faces, we have $F=\bigcap_{i} F_{i}$ is a face, and

$$
\left(\bigcap_{i} F_{i}\right)^{\prime}=\bigcup_{i} F_{i}^{\prime}
$$

Proposition 3.2. Let $F$ be a face of the state space of a pseudo effect algebra $E$ with $(\mathrm{RDP})$ and let $\mathcal{S}(E) \neq \emptyset$. Then its complementary face $F^{\prime}$ consists of all states $s^{\prime} \in \mathcal{S}(E)$ such that $s^{\prime} \wedge s=0$ for every state $s \in F$.

Equivalently, $F^{\prime}$ consists of all states $s^{\prime} \in \mathcal{S}(E)$ such that if $s \in F$ is such that $\alpha s \leq^{+} s^{\prime}$ for some constant $\alpha \geq 0$, then $\alpha=0$.

Proof. We know that $\mathcal{S}(E)$ is a simplex. According to [Goo, Prop 10.12], $F^{\prime}$ is the union of all faces of $\mathcal{S}(E)$ that are disjoint from $F$. If $s^{\prime} \in F^{\prime}$ and $s \in F$, and if $s^{\prime}$ and $s$ belong to mutually disjoint faces, then $s^{\prime} \wedge s=0$, see [Dvu3. Prop 4.2]. Conversely, let $s^{\prime}$ be a state on $E$ such that $s^{\prime} \wedge s=0$ for each $s \in F$. Then by Dvu3, Prop 4.2], $s^{\prime}$ and $s$ belong to mutually disjoint faces. Let $F\left(s^{\prime}\right)$ and $F(s)$ be the faces generated by $s^{\prime}$ and $s$. Therefore, $F\left(s^{\prime}\right) \cap F(s)=\emptyset$. But $F=\bigcup_{s \in F} F(s)$, so that $F\left(s^{\prime}\right) \cap F=\bigcup_{s \in F} F\left(s^{\prime}\right) \cap F(s)=\emptyset$ that gives $s^{\prime} \in F^{\prime}$

Now let $s^{\prime} \in F^{\prime}$ and let $s \in F$ be such that $\alpha s \leq s^{\prime}$ for some $\alpha \geq 0$. If $\alpha>0$, then $s \leq s^{\prime} / \alpha$ that $s$ belongs to the face generated by $s^{\prime}$ that is impossible, whence $\alpha=0$.

Assume that $F_{0}$ is the set of all those states $s^{\prime}$ on $E$ such that if $\alpha s \leq+s^{\prime}$ for $s \in F$ and $\alpha \geq 0$, then $\alpha=0$. We have seen that $F^{\prime} \subseteq F_{0}$. Let $s^{\prime} \in F_{0}$ and $s \in F$ If $s^{\prime} \wedge s>^{+} 0$, then $s$ belongs to the face generated by $s^{\prime}$, hence, $s \leq^{+} t s^{\prime}$ for some $t>0$. This gives $s / t \leq+s$ so that $1 / t=0$ that is absurd. Hence, $F_{0} \subseteq F^{\prime}$.

Let $K_{1}, \ldots, K_{n}$ be convex subsets of $K$. We say that $K$ is the direct convex sum of $K_{1}, \ldots, K_{n}$ if (i) $K$ equals the convex hull of $\bigcup_{i=1}^{n} K_{i}$ and every element $x \in K$ can be uniquely expressed as a convex combination of some elements $x_{i} \in K_{i}$, $i=1, \ldots, n$. That is, if

$$
\alpha_{1} x_{1}+\cdots+\alpha_{n} x_{n}=\beta_{1} y_{1}+\cdots+\beta_{n} y_{n}
$$

are convex combinations of $x_{i}, y_{i} \in K_{i}$, for $i=1, \ldots, n$, then $\alpha_{i}=\beta_{i}$ for all $i$ 's and $x_{i}=y_{j}$ for those $i$ such that $\alpha_{i}>0$.

Theorem 3.3. Let $E$ be a pseudo effect algebra with (RDP) and let $\mathcal{S}(E) \neq \emptyset$. Let $I$ be a normal ideal of $E$, and set

$$
F=\{s \in \mathcal{S}(E): I \subseteq \operatorname{Ker}(s)\} .
$$

Then $F$ is a closed face of $\mathcal{S}(E)$, and $\mathcal{S}(E)$ is the direct convex sum of $F$ and its complementary face. 
Proof. By Proposition 3.1, $F$ is a closed face of $\mathcal{S}(E)$. If $F$ is empty, then $F^{\prime}=\mathcal{S}(E)$.

Now assume $F$ is non-void. Let $\mathcal{J}(E)$ be the set of all Jordan signed measures on $E$. By Theorem 2.1, $\mathcal{J}(E)$ is an Abelian Dedekind complete $\ell$-group, and $\mathcal{S}(E)$ is a base for the positive cone $\mathcal{J}(E)^{+}=\{\alpha s: \alpha \geq 0, s \in \mathcal{S}(E)\}$. We show that the set

$$
C=\{\alpha s: \alpha \geq 0, s \in \mathcal{S}(E), s \in F\}
$$

contains the supremum of any subset of $C$ which is bounded above in $\mathcal{J}(E)$. Given a nonempty system of positive measures $\left\{m_{i}\right\}_{i}$ from $C$ that is bounded in $\mathcal{J}(E)$, let $m=\bigvee_{i} m_{i}$. According to Theorem 2.1.

$$
m(x)=\sup \left\{d\left(x_{1}\right)+\cdots+d\left(x_{n}\right): x=x_{1}+\cdots+x_{n}\right\}, \quad x \in E,
$$

where $d(x)=\sup _{i} m_{i}(x), x \in E$.

If $x \in I$, then $x=x_{1}+\cdots+x_{n}$ entails $x_{1}, \ldots, x_{n} \in I$ so that $d\left(x_{j}\right)=$ $\sup _{i} m_{i}\left(x_{j}\right)=0$ for each $j=1, \ldots, n$. Hence, $m(x)=0$ and $m \in C$.

According to [Goo, Prop 10.14], $\mathcal{S}(E)$ is the direct convex sum of $F$ and its complementary face.

Let $F$ be a face of $\mathcal{S}(E)$, where $E$ is a pseudo effect algebra with (RDP). We set

$$
V(F):=\{\alpha s: \alpha \geq 0, s \in F\} .
$$

It is a cone that is a subcone of $\mathcal{M}^{+}(S)$, i.e., if (i) $m_{1}, m_{2} \in V(F)$, then $m_{1}+m_{2} \in$ $V(F)$, (ii) $\mathbb{R}^{+}(F) \subseteq V(F)$ (ii) $-V(F) \cap V(F)=\{0\}$. We say that $V(F)$ is $\vee$-closed if, for any chain $\left\{m_{i}\right\}$ from $V(F)$ bounded in $\mathcal{M}^{+}(E), \bigvee_{i} m_{i} \in V(F)$.

If $F^{\prime}$ is the complementary face of $F$, according to Proposition 3.2, $m \in V\left(F^{\prime}\right)$ iff for any $t \in V(F)$ with $t \leq^{+} m$, we have $t=0$.

For an arbitrary cone $V$ of $\mathcal{S}(E)$, we denote by $V^{\sharp}$ the set of all those measures $t \in \mathcal{M}^{+}(E)$ that $m \leq^{+} t$ for $m \in V$ entails $m=0$. The elements of $V^{\sharp}$ are said to be $V$-singular measures. Hence, $V(F)^{\sharp}=V\left(F^{\prime}\right)$ for any face $F$ of $\mathcal{S}(E)$ of a pseudo effect algebra $E$ with (RDP).

Theorem 3.4. Let $F$ be a face of the state space $\mathcal{S}(E)$ of a pseudo effect algebra $E$ with (RDP). If $V(F)$ is $\vee$-closed, then $\mathcal{S}(E)$ equals the direct convex sum of $F$ and its complementary face $F^{\prime}$.

In addition, every measure $m$ on $E$ can be uniquely decomposed as a sum

$$
m=m_{1}+m_{1}
$$

of two measures such that $m_{1} \in V(F)$ and $m_{2} \in V\left(F^{\prime}\right)$.

Proof. Existence: Let $m$ be a measure on $E$, and let $\Gamma(m)=\left\{m_{1} \in V(F): m_{1} \leq^{+}\right.$ $m\}$. Since the zero measure belongs to $\Gamma(m), \Gamma(m)$ is non-void. Let $\left\{m_{i}\right\}$ be a chain of elements from $\Gamma(m)$. The measure $m$ is an upper bound for $\left\{m_{i}\right\}$. By Theorem 2.1, $m_{0}:=\bigvee_{i} m_{i}$ is a measure on $E$ and by the hypotheses, $m_{0}$ belongs to $V(F)$. It follows from the Zorn's Lemma that $\Gamma(m)$ contains a maximal element $m_{1}$ such that $m_{1} \leq^{+} m$.

If we set $m_{2}=m-m_{1}$, we show that $m_{2} \in V\left(F^{\prime}\right)$. Let $t \in V(F)$ and let $t \leq^{+} m_{2}$. Then $m_{1}+t \leq^{+} m_{1}+m_{2}=m$. Since $m_{1}+t \in V(F)$, the maximality of $m_{1}$ in $\Gamma(m)$ implies $t=0$, and therefore, $m_{2} \in V(F)$. 
Uniqueness: Now let $s$ be an arbitrary state on $E$. According to the first part of the present proof, we see that $s$ can be decomposed in the convex form

$$
s=\lambda_{1} s_{1}+\lambda_{2} s_{2},
$$

where $s_{1} \in F$ and $s_{2} \in F^{\prime}$. This implies that $\mathcal{S}(E)$ is a direct convex sum of $F$ and $F^{\prime}$. In particular, according to Goo, Prop 10.12], this yields that the decomposition (3.2) is unique, i.e. if $s=\alpha_{1} s_{1}^{\prime}+\alpha_{2} s_{2}^{\prime}$ is another convex combination of $s_{1}^{\prime} \in F$ and $s_{2}^{\prime} \in F^{\prime}$, then $\alpha_{i}=\lambda_{i}$ for $i=1,2$, and if $\alpha_{i}>0$ implies $s_{i}=s_{i}^{\prime}$. In particular, this implies that the decomposition in (3.1) is unique.

\section{Decomposition of States on Pseudo Effect Algebras}

This section presents the main results of the paper: Yosida-Hewitt type and Lebesgue type of decomposition of finitely additive measures and states.

Using closed faces $F$, we can decompose any state $s$ on a pseudo effect algebra with (RDP) in the unique form: $s=\lambda s_{1}+(1-\lambda) s_{2}$, where $s_{1} \in F$ and $s_{2} \in F^{\prime}$, where $F^{\prime}$ is the complementary face of $F$, see [Goo, Thm 11.28]. But not every face of $\mathcal{S}(E)$ is closed. E.g. the set of all $\sigma$-additive states on $E$ is a face that is not necessarily closed. However, also for some such situations we show that $\mathcal{S}(E)$ can be the direct convex sum of the face $F$ and its complementary face.

A non-empty set $X$ of a poset $E$ is directed downwards (directed upwards), and we write $D \downarrow(D \uparrow)$, if for any $x, y \in X$ there exists $z \in D$ such that $z \leq x, z \leq y$ $(z \geq x, z \geq y)$. Two downwards directed sets $\left\{x_{t}: t \in T\right\}$ and $\left\{y_{t}: t \in T\right\}$ indexed by the same index set $T$ are called downwards equidirected if, for any $s, t \in T$, there exists $v \in T$ such that $x_{v} \leq x_{s}$ and $x_{v} \leq x_{t}$ as well as $y_{v} \leq y_{s}$ and $y_{v} \leq y_{t}$. A similar definition holds for upwards directed sets.

Let $x \in E$ and $D \subseteq E$. We say that $D \uparrow x$ if $D \uparrow$ and $x=\bigvee D$. Dually we define $D \downarrow x$, i.e. $D \downarrow$ and $x=\bigwedge D$.

Let $\left\{a_{n}\right\}$ be a sequence of elements of a pseudo effect $E$ such that $b_{n}=a_{1}+\cdots+a_{n}$ exists in $E$ for each $n \geq 1$ and if $a=\bigvee_{n} b_{n}$ exists in $E$, we write $a=\sum_{n} a_{n}$.

A signed measure $m$ on a pseudo effect algebra $E$ is $\sigma$-additive if, $\left\{a_{n}\right\} \nearrow a$, i.e. $a_{n} \leq a_{n+1}$ for each $n \geq 1$ and $\bigvee_{n} a_{n}=a$, then $m(a)=\lim _{n} m\left(a_{n}\right)$. A signed measure $m$ is $\sigma$-additive iff $\left\{a_{n}\right\} \searrow 0$ entails $\lim _{n} m\left(a_{n}\right)=0$.

Now let $E$ be an effect algebra (not a pseudo effect algebra). We say that a system $\left\{a_{t}\right\}_{t \in T}$ of elements of $E$ is summable if, for each finite subset $F \subseteq T$, the element $a_{F}=\sum_{t \in F} a_{t}$ is defined in $E$. If there exists the element $a=\bigvee\left\{a_{F}: F \subseteq T\right\}$, we called it the sum of the summable system $\left\{a_{t}\right\}_{t \in T}$, and write $a=\sum_{t \in T} a_{t}$.

A signed measure $m$ on an effect algebra is said to be completely additive if $m(a)=\sum_{t \in T} m\left(a_{t}\right)$ whenever $a=\sum_{t \in T} a_{t}$.

We denote by $\mathcal{J}(E)_{\sigma}, \mathcal{J}(E)_{\text {ca }}$ the sets of all $\sigma$-additive Jordan signed measures and completely additive Jordan signed measures on $E$, respectively. In the same way we define $\mathcal{M}^{+}(E)_{\sigma}, \mathcal{M}^{+}(E)_{\text {ca }}$ and similarly for the states: $\mathcal{S}(E)_{\sigma}$ and $\mathcal{S}(E)_{\text {ca }}$ denote the systems of all $\sigma$-additive and completely additive states on $E$.

Then $\mathcal{S}(E)_{\text {ca }} \subseteq \mathcal{S}(E)_{\sigma} \subseteq \mathcal{S}(E)$. Each of these sets can be empty. Moreover, $\mathcal{S}(E)_{\sigma}$ and $\mathcal{S}(E)_{\text {ca }}$ are also faces of $\mathcal{S}(E)$, and

$$
V\left(\mathcal{S}(E)_{\sigma}\right)=\mathcal{M}^{+}(E)_{\sigma} \quad \text { and } \quad V\left(\mathcal{S}(E)_{\mathrm{ca}}\right)=\mathcal{M}^{+}(E)_{\mathrm{ca}} .
$$

Proposition 4.1. Let $m_{1}, \ldots, m_{n}$ be completely additive measures on an effect algebra $E$ that satisfies (RDP). Then $m=m_{1} \vee \cdots \vee m_{n}$ is also a completely additive measure on $E$. 
The same is true if $m_{1}, \ldots, m_{n}$ are $\sigma$-additive measures on a pseudo effect algebra $E$ with (RDP).

Proof. Without loss of generality, we can assume that $n=2$. Let $a=\sum_{t \in T} a_{t}$ and let $F$ be any finite subset of $T$ and let $a_{F}=\sum_{t \in F} a_{t}$. Given $\epsilon>0$, there is $F_{0}$ such that, for each finite $F \supseteq F_{0}, m_{1}\left(a-a_{F}\right)<\epsilon / 2$ and $m_{2}\left(a-a_{F}\right)<\epsilon / 2$.

By Theorem 2.1. $m\left(a-a_{F}\right)=\sup \left\{m_{1}(x)+m_{2}(y): x+y=a-a_{F}\right\}$. Since $x \leq a-a_{F}$ and $y \leq a-a_{F}$, we have $m_{1}(x) \leq m_{1}\left(a-a_{F}\right)<\epsilon / 2$ and $m_{2}(y) \leq$ $m_{2}\left(a-a_{F}\right)<\epsilon / 2$. Then $m_{1}(x)+m_{2}(y)<\epsilon$, consequently $m\left(a-a_{F}\right) \leq \epsilon$. Hence, $m(a)-m\left(a_{F}\right) \leq \epsilon$ and $m$ is completely additive.

Lemma 4.2. If $\left\{f_{t}\right\}_{t \in T} \uparrow f$ and $\left\{g_{t}\right\}_{t \in T} \uparrow g$, where $f_{t}, g_{t}, f, g \in \mathbb{R}^{+}$for all $t \in T$. Then

$$
\bigvee_{s, t}\left(f_{s}+g_{t}\right)=f+g
$$

If, in addition, $\left\{f_{t}\right\}_{t \in T}$ and $\left\{g_{t}\right\}_{t \in T}$ are upwards equidirected, then

$$
\left\{f_{t}+g_{t}\right\}_{t \in T} \uparrow f+g .
$$

Proof. We have $f_{s}+g_{t} \leq f+g$ for all $s, t \in T$. If $f_{s}+g_{t} \leq x$ for some $x \in \mathbb{R}$, then $f_{s} \leq x-g_{t}$ so that $f \leq x-g_{t}$ and hence, $g_{t} \leq x-f$ so that $g \leq x-f$ and $f+g \leq x$ that gives, (4.2).

Now assume $\left\{f_{t}\right\}$ and $\left\{g_{t}\right\}$ are upwards equidirected. It is clear that $f_{t}+g_{t} \leq$ $f+g$. The equidirectness entails that, for all indices $s_{0}, t_{0} \in T$, there exists an index $t$ such that $f_{s_{0}} \leq f_{t}$ and $f_{t_{0}} \leq g_{t}$. Therefore, for all indices $s_{0}$ and $t_{0}$, $f_{s_{0}}+g_{t_{0}} \leq f_{t}+g_{t} \leq f+g$ which by (4.2) gives (4.3).

Lemma 4.3. Let $E$ be a pseudo effect algebra with (RDP). If $\left\{m_{i}\right\}$ is a chain of measures in $\mathcal{M}^{+}(E)$ that is bounded above, then for $m_{0}=\bigvee_{i} m_{i}$ we have

$$
m_{0}(a)=\sup _{i} m_{i}(a)
$$

for each $a \in E$.

Proof. We assert that if $d(x)=\bigvee_{i} m_{i}(x), x \in E$, then $d$ is additive, i.e., $d(x+$ $y)=d(x)+d(y)$ whenever $x+y$ is defined in $E$. This follows from the fact that $\left\{m_{i}(x)\right\} \uparrow d(x)$ and $\left\{m_{i}(y)\right\} \uparrow d(y)$, are upwards equidirected because $\left\{m_{i}\right\}$ is a chain, and $\left\{m_{i}(x)+m_{i}(y)\right\}=\left\{m_{i}(x+y)\right\} \uparrow(d(x)+d(y))$ by (4.3).

Since, $d$ is a measure such that $m_{i} \leq^{+} d \leq+m_{0}$, we conclude $d=m_{0}$.

Now we present a Yosida-Hewitt type of decomposition for measures on $E$.

Theorem 4.4. Let $E$ be an effect algebra with (RDP). Then every measure $m$ on $E$ can be uniquely expressed in the form

$$
m=m_{1}+m_{2},
$$

where $m_{1} \in \mathcal{M}^{+}(E)_{\text {ca }}$ and $m_{2}$ is a finitely additive measure on $E$ such that if $t \in \mathcal{M}^{+}(E)_{\text {ca }}$, such $t \leq{ }^{+} m_{2}$, then $t=0$.

In particular, every state $s$ on $E$ can be uniquely expressed as a convex combination

$$
s=\lambda_{1} s_{1}+\lambda_{2} s_{2}
$$

where $s_{1}$ is a completely additive state and $s_{2}$ is a finitely additive state such that if $\alpha s^{\prime} \leq+s_{2}$ for some completely additive state $s^{\prime}$ on $E$ and for some constant $\alpha \geq 0$, then $\alpha=0$. 
Proof. First we show that $\mathcal{M}^{+}(E)_{\text {ca }}$ is a $\vee$-closed cone in $\mathcal{M}^{+}(E)$. Thus let $\left\{m_{i}\right\}$ be a chain of completely additive measures on $E$ that is bounded above by a finitely additive measure $m^{\prime}$. By Theorem 2.1 there is $m_{0}=\bigvee_{i} m_{i}$ that is finitely additive and $m_{0} \leq^{+} m^{\prime}$.

We assert that $m_{0}$ is completely additive. Let $a=\sum_{t \in T} a_{t}$ exist in $L$. Then from the monotonicity of $m_{0}$ we have $m_{0}\left(a_{F}\right) \leq m_{0}(a)$, where $a_{F}:=\sum_{t \in F} a_{t}$ for any $F$ finite subset of the index set $T$.

Assume that $m_{0}\left(a_{F}\right) \leq x$ for some $x \in \mathbb{R}^{+}$and for any finite $F$. Then $m_{i}\left(a_{F}\right) \leq x$ for any $i$ and any $F$. The complete additivity of $m_{i}$ entails that $m_{i}(a) \leq x$ for any $i$, so that by $(4.4), m_{0}(a)=\sup _{i} m_{i}(a) \leq x$. This gives

$$
m_{0}(a)=\sup _{F} \sum_{t \in F} m_{0}\left(a_{t}\right)
$$

consequently $m_{0} \in \mathcal{M}^{+}(E)_{\text {ca }}$, and $\mathcal{M}^{+}(E)_{\text {ca }}$ is a $\vee$-closed cone of $\mathcal{J}(E)$.

Now let $m$ be an arbitrary finitely additive measure on $E$. Since all the conditions of Theorem 3.4 are satisfied, we obtain the unique decomposition $m=m_{1}+m_{2}$ in question. Similarly we have (4.6).

Theorem 4.5. Let $E$ be a pseudo effect algebra with (RDP). Then every measure $m$ on $E$ can be uniquely expressed in the form

$$
m=m_{1}+m_{2},
$$

where $m_{1} \in \mathcal{M}^{+}(E)_{\sigma}$ and $m_{2}$ is a finitely additive measure on $E$ such that if $t \in \mathcal{M}^{+}(E)_{\sigma}, t \geq 0$, such $t \leq^{+} m_{2}$, then $t=0$.

In particular, every state $s$ on $E$ can be uniquely expressed as a convex combination

$$
s=\lambda_{1} s_{1}+\lambda_{2} s_{2}
$$

where $s_{1}$ is a $\sigma$-additive state and $s_{2}$ is a finitely additive state such that if $\alpha s^{\prime} \leq^{+} s_{2}$ for some completely additive state $s^{\prime}$ on $E$ and for some constant $\alpha \geq 0$, then $\alpha=0$.

Proof. It follows the same steps as the proof of Theorem 4.4.

Remark 4.6. Theorems 4.5 and 4.5 have been proved in [11, 12, 9, 17]. They are analogues of the classical Yosida-Hewitt decomposition from $\mathrm{YoHe}$. In DeMo, the component $m_{2}$ from Theorem 4.4 is said to be a weakly purely additive measure and that from Theorem 4.5 a purely additive measure.

Now we present another Yosida-Hewitt type decomposition for an analogue of complete additivity of measures for pseudo effect algebra. We say that a measure $m$ on $E$ is upwards continuous if $\left\{a_{t}\right\} \uparrow a$ entails $\left\{m\left(a_{t}\right)\right\} \uparrow m(a)$. A measure $m$ is upwards continuous iff $\left\{a_{t}\right\} \downarrow 0$ implies $\left\{m\left(a_{t}\right)\right\} \downarrow 0$.

For example, if $E$ is an effect algebra, then $m$ is completely additive whenever $m$ is upwards continuous. Indeed, let $m$ be an upwards continuous measure and let $a=\sum_{t \in T} a_{t}$. Given any finite subset $F$ of indices we define $a_{F}=\sum_{t \in F} a_{t}$. Then $\left\{a_{F}\right\}_{F}$ is upwards directed and $\left\{a_{F}\right\} \uparrow a$, so that $m(a)=\sum_{t} m\left(a_{t}\right)$.

Theorem 4.7. Let $E$ be a pseudo effect algebra with (RDP). Then every measure $m$ on $E$ can be uniquely expressed in the form

$$
m=m_{1}+m_{2},
$$

where $m_{1}$ is an upwards continuous measure and $m_{2}$ is a finitely additive measure on $E$ such that if $t$ is an upwards continuous measure with $t \leq^{+} m_{2}$, then $t=0$. 
In particular, every state $s$ on $E$ can be uniquely expressed as a convex combination

$$
s=\lambda_{1} s_{1}+\lambda_{2} s_{2},
$$

where $s_{1}$ is an upwards continuous state and $s_{2}$ is a finitely additive state such that if $\alpha s^{\prime} \leq^{+} s_{2}$ for some upwards continuous state $s^{\prime}$ on $E$ and for some constant $\alpha \geq 0$, then $\alpha=0$.

Proof. Let $\mathcal{M}^{+}(E)_{\mathrm{uc}}$ and $\mathcal{S}(E)_{\mathrm{uc}}$ be the sets of upwards continuous measures and states, respectively, on $E$. Then $\mathcal{S}(E)_{\mathrm{uc}}$ is a face and $V\left(\mathcal{S}(E)_{\mathrm{uc}}\right)=\mathcal{M}^{+}(E)$. We show that $\mathcal{M}^{+}(E)_{\mathrm{uc}}$ is a $\vee$-closed cone. Indeed, let $\left\{m_{i}\right\}$ be a chain in $\mathcal{M}^{+}(E)_{\mathrm{uc}}$. Then $\sup _{t} m\left(a_{t}\right) \leq m(a)$. By (4.4), for $m=\bigvee_{i} m_{i}$, we have $m(a)=\sup _{i} m_{i}(a)$ for each $a \in E$. Assume $\left\{a_{t}\right\} \uparrow a$. Given $\epsilon>0$, there is $i$ such that $m_{i}(a)>m(a)-\epsilon / 2$. Since $m_{i}$ is upwards continuous, there is $a_{t}$ such that $m_{i}\left(a_{t}\right)>m_{i}(a)-\epsilon / 2$. Then $m_{i}\left(a_{t}\right) \geq m(a)-\epsilon$ and by (4.4), $m\left(a_{t}\right)>m(a)-\epsilon$. Therefore, $m(a)=\sup _{t} m\left(a_{t}\right)$.

For the final desired result, we apply Theorem 3.4

In what follows, we present two types of the Lebesgue decomposition.

Let $m_{1}$ and $m_{2}$ be measures on a pseudo effect algebra $E$. We say that (i) $m_{1}$ is absolutely continuous with respect to $m_{2}$, and we write $m_{1} \ll m_{2}$ if $m_{2}(a)=0$ implies $m_{1}(a)=0$ for $a \in E$. (ii) $m_{1}$ is $m_{2}$-continuous, and we write $m_{1} \ll_{\epsilon} m_{2}$ provided given $\epsilon>0$, there is a $\delta>0$ such that $m_{2}(a)<\delta$ yields $m_{1}(a)<\epsilon$. (iii) $m_{1} \perp m_{2}$ if there is an element $a \in E$ such that $m_{2}(a)=0=m_{1}\left(a^{-}\right)$.

It is clear that $m_{1} \ll_{\epsilon} m_{2}$ entails $m_{1} \ll m_{2}$.

Theorem 4.8. Let $E$ be a pseudo effect algebra with (RDP). Let t be a fixed measure on $E$. Then every measure $m$ on $E$ can be uniquely expressed in the form

$$
m=m_{1}+m_{2},
$$

where $m_{1}$ and $m_{2}$ are finitely additive measures on $E$ such that $m_{1} \ll_{\epsilon} t$ and if $m^{\prime}$ is any measure such that $m^{\prime} \ll_{\epsilon} t$ and $m_{1} \leq^{+} m_{2}$, then $m^{\prime}=0$. Moreover, $m_{2} \wedge t=0$.

In particular, every state $s$ on $E$ can be uniquely expressed as a convex combination of two states $s_{1}$ and $s_{1}$ on $E$,

$$
s=\lambda_{1} s_{1}+\lambda_{2} s_{2},
$$

where $s_{1} \ll_{\epsilon} t$ and if $s^{\prime}$ is any state such that $s^{\prime} \ll_{\epsilon} t$ and $\alpha s^{\prime} \leq^{+} s_{2}$, then $\alpha=0$.

Proof. If $t=0$, the statement is trivial. Suppose that $t(1)>0$ and let $F(t)=\{s \in$ $\left.\mathcal{S}(E): s \ll_{\epsilon} t\right\}$. Then $t_{0}=t / t(1) \in F(t)$ and $F(t)$ is a non-empty face of $\mathcal{S}(E)$.

Let $C(t):=\left\{s \in \mathcal{M}^{+}(E): s \ll_{\epsilon} t\right\}$. Then $C(t)$ is a nonempty cone that is a subcone of $\mathcal{M}^{+}(E)$, and $C(t)=V(F(t))$. We claim that $C(t)$ is $\vee$-closed. Let $\left\{m_{i}\right\}$ be a chain from $C(t)$ that is bounded above by $m^{\prime} \in \mathcal{M}^{+}(E)$. If we define $d(x)=$ $\bigvee_{i} m_{i}(x)$, according to Lemma 4.3, we have $d=m_{0}:=\bigvee_{i} m_{i}$ and $\left\{m_{i}(1)\right\} \uparrow d(1)$ Since $\mathcal{J}(E)$ is an Abelian Dedekind complete $\ell$-group, we have $\left\{m_{0}-m_{i}\right\} \downarrow 0$. Therefore, $\left\{\left(m_{0}-m_{i}\right)(1)\right\}=\left\{m_{0}(1)-m_{i}(1)\right\} \downarrow 0$. Thus given $\epsilon>0$, there is an index $i_{0}$ such that, for each $m_{i}$ with $m_{i} \geq^{+} m_{i_{0}}$, we have $m_{0}(1)-m_{i}(1)<\epsilon / 2$.

Fix the index $i_{0}$. Given $\epsilon>0$, there is $\delta>0$ such that $t(a)<\delta$ implies $m_{i_{0}}(a)<$ $\epsilon / 2$.

Calculate, 
$m_{0}(a)=m_{i_{0}}(a)+\left(m_{0}-m_{i_{0}}\right)(a) \leq m_{i_{0}}(a)+\left(m_{0}-m_{i_{0}}\right)(1) \leq \epsilon / 2+\left(m_{0}-m_{i_{0}}\right)(1)<\epsilon$ that gives $m_{0} \ll_{\epsilon} t$ and $m_{0} \in C(t)$.

Applying the general result from Theorem 3.4 we have the existence and uniqueness of (4.9).

Now we show that $m_{2} \wedge t=0$. Let $k$ be a measure on $E$ such that $k \leq^{+} m_{2}$ and $k \leq^{+} t$. Then $k \ll_{\epsilon} t$, so that $k=0$ and whence $m_{2} \wedge t=0$.

Theorem 4.9. Let $E$ be a pseudo effect algebra with (RDP). Let $t$ be a fixed measure on $E$. Then every measure $m$ on $E$ can be uniquely expressed in the form

$$
m=m_{1}+m_{2},
$$

where $m_{1}$ and $m_{2}$ are finitely additive measures on $E$ such $m_{1} \ll t$ and if $m^{\prime}$ is any measure on $E$ such that $m^{\prime} \ll t$ and $m^{\prime} \leq^{+} m_{2}$, then $m^{\prime}=0$.

Proof. Let us define $V(t)=\left\{m \in \mathcal{M}^{+}(E): m \ll t\right\}$ and $C(t):=\{s \in \mathcal{S}(E)$ : $s \ll t\}$. Then $F(t)$ is a face of $\mathcal{S}(E)$ and it generates $V(t)=V(F(t))$. Assume that $\left\{m_{i}\right\}$ is a chain from $V(t)$ that is bounded in $\mathcal{J}(E)$. If we set $d(x)=\bigvee_{i} m_{i}(x)$ for each $x \in E$, then according to (4.4), $d=m_{0}:=\bigvee_{i} m_{i}$. Therefore, if $t(a)=0$, then $m_{i}(a)=0$ for each $i$ so that $m(a)=d(a)=0$ and $m_{0} \in V(t)$.

The existence and uniqueness of (4.11) follows from Theorem 3.4.

Finally, we show a relation among two types of continuity of measures.

Proposition 4.10. Let $s_{1}$ and $s_{s}$ be two $\sigma$-additive measures on a $\sigma$-complete $M V$-algebra E. Then $s_{1} \ll s_{2}$ if and only if $s_{1} \ll_{\epsilon} s_{2}$.

Proof. It is clear that $s_{1} \ll{ }_{\epsilon} s_{2}$ entails $s_{1} \ll s_{2}$. Now let us suppose $s_{1} \ll s_{2}$ and let (ad absurdum) $s_{1} K_{\epsilon} s_{2}$. Then there is an $\epsilon>0$ such that for each $n \geq 1$, there is an $a_{n} \in E$ such that $s_{2}\left(a_{n}\right)<1 / 2^{n}$ and $s_{1}\left(a_{n}\right) \geq \epsilon$. Set $a=\bigwedge_{n=1}^{\infty} \bigvee_{n=k}^{\infty} a_{k}$. Then

$$
s_{2}(a) \leq s_{2}\left(a_{n} \vee a_{n+1} \vee \cdots\right) \leq \sum_{k=n}^{\infty} s_{2}\left(a_{k}\right)<1 / 2^{n-1},
$$

so that $s_{2}(a)=0$.

On the other hand, $s_{1}(a)=\lim _{n} s_{1}\left(a_{n} \vee a_{n+1} \vee \cdots\right) \geq \lim \sup _{n} s_{1}\left(a_{n}\right) \geq \epsilon$ that contradicts $s_{1} \ll s_{2}$.

We say that a measure $t$ on $E$ is Jauch-Piron if $t(a)=t(b)=0$ entails there is an element $c \in E$ such that $a, b \leq c$ and $m(c)$. Every measure on an MV-algebra is Jauch-Piron.

Let $E$ be a pseudo effect with (RDP). According to [Dvu, Thm 3.2], we say that an element $a \in E$ is said to be central or Boolean, if $a \wedge a^{-}=0$. Then also $a \wedge a^{\sim}=0$ and $a^{-}=a^{\sim}$. Moreover, for any $x \in E, x \wedge a$ is defined in $E$, and

$$
x=(x \wedge a)+\left(x \wedge a^{-}\right) .
$$

Let $C(E)$ be the set of all central elements of $E$. Then it is a Boolean algebra that is a subalgebra of $E$. If, in addition, $E$ is monotone $\sigma$-complete, then $C(E)$ is a Boolean $\sigma$-algebra, $\mathrm{Dvu}$, Thm 5.11]. We recall that a pseudo effect algebra $E$ is said to be monotone $\sigma$-complete provided, for any sequence $\left\{a_{n}\right\}$ from $E$ such that $a_{n} \leq a_{n+1}$ for each $n \geq 1, a=\bigvee_{n} a_{n}$ is defined in $E$. 
If $a, b$ are central elements and $t$ is a measure, then $t(a)+t(b)=t(a \wedge b)+t(a \vee b)$, so that if, in addition, $t(a)=t(b)=0$, then $t(a \vee b)=0$.

Now we present another Lebesgue type of decomposition for measures. For two measures $m$ and $t$ on $E$ we write $m \ll_{C} t$ provided $t(a)=0$ for $a \in C(E)$ implies $m(a)=0$. It is a weaker form of $m \ll t$.

Theorem 4.11. Let $E$ be a monotone $\sigma$-complete pseudo effect algebra with (RDP) and let $t$ be a $\sigma$-additive measure on $E$. Every $\sigma$-additive measure $m$ on $E$ can be uniquely decomposed in the form

$$
m=m_{1}+m_{2}
$$

such that $m_{1}, m_{2}$ are $\sigma$-additive measures, $m_{1} \ll_{C} t$, and $m_{2} \perp t$.

In particular, every $\sigma$-additive measure $s$ can be uniquely decompose in the form

$$
s=\lambda s_{1}+(1-\lambda) s_{2},
$$

where $s_{1}$ and $s_{2}$ are $\sigma$-additive measures on $E$ such that $s_{1} \ll t$ and $s_{2} \perp t$.

Proof. Existence: Let $\operatorname{Ker}(t)_{C}=\{a \in C(E): t(a)=0\}$. The zero element 0 is central, so that $0 \in \operatorname{Ker}(t)_{C}$. We order the elements of $\operatorname{Ker}(t)_{C}$ by $a \preceq b$ iff $a, b \in \operatorname{Ker}(t)_{C}$ and $m(a) \leq m(b)$. Then $\preceq$ is a partial order and now let $\left\{a_{i}\right\}$ be a chain of elements from $\operatorname{Ker}(t)_{C}$ with respect to $\preceq$, and let $\delta=\sup _{i} m\left(a_{i}\right)$. Then either there is an upper bound $a$ of $\left\{a_{i}\right\}$ itself or there is a sequence $\left\{a_{n}\right\}$ in $\left\{a_{i}\right\}$ such that $m\left(a_{n}\right)<m\left(a_{n+1}\right) \nearrow \delta$. Set $a=\bigvee_{n} a_{n}$; then $a \in \operatorname{Ker}(t)_{C}$ and $m(a)=\lim _{n} m\left(a_{n}\right)=\delta$, so that $a$ is an upper bound in $\operatorname{Ker}(t)_{C}$ for the chain $\left\{a_{i}\right\}$. Applying the Zorn Lemma, $\operatorname{Ker}(t)_{C}$ contains a maximal element, say $a_{0}$.

Set $m_{1}(x)=m\left(x \wedge a_{0}^{-}\right)$and $m_{2}(x)=m\left(x \wedge a_{0}\right)$ for each $x \in E$. Since $C(E)$ is a Boolean $\sigma$-algebra, and $x=\left(x \wedge a_{0}^{-}\right)+\left(x \wedge a_{0}\right)$ for each $x \in E$, we see that $m_{1}$ and $m_{2}$ are $\sigma$-additive measures on $E$ such that $m=m_{1}+m_{2}$. Since $t\left(a_{0}\right)=0$, then $m_{2}\left(a_{0}^{-}\right)=m\left(a_{0}^{-} \wedge a_{0}\right)=0$ so that $m_{2} \perp t$. We assert that $m_{1} \ll_{C} t$. If not, there is an element $a \in \operatorname{Ker}(t)_{C}$ such that $m_{1}(a)=m\left(a \wedge a_{0}^{-}\right)>0$. Since $a_{0} \leq a_{0} \vee\left(a \wedge a_{0}^{-}\right) \in \operatorname{Ker}(t)_{C}$ which contradicts the maximality of $a_{0}$. This proves that $m_{1} \ll_{C} t$.

Uniqueness: Let $F(t)_{\sigma}$ be the set of all $\sigma$-additive states $s$ on $E$ such that $s \ll_{C} t$. Then $F(t)_{\sigma}$ is a face and due to Theorems 4.4 4.5, if $\left\{m_{i}\right\}$ is a bounded chain from $V\left(F(t)_{\sigma}\right)$, then $m_{0}=\bigvee_{i} m_{i}$ is a $\sigma$-additive measure, and in view of (4.4), $m_{0} \ll_{C} t$. This gives that $V\left(F(t)_{\sigma}\right)$ is a $\vee$-closed cone. Now let $m^{\prime}$ be an arbitrary $\sigma$-additive measure from $V\left(F(t)_{\sigma}\right)$ such that $m^{\prime} \leq^{+} m_{2}$. Then $m^{\prime}\left(a_{0}\right)=0$ while $t\left(a_{0}\right)$ and $m^{\prime}\left(a_{0}^{-}\right) \leq m_{1}\left(a_{0}^{-}\right)=0$ so that $m^{\prime}=0$. Therefore, $m_{1} \in V\left(F(t)_{\sigma}^{\prime}\right)$ so that by Theorem 3.4 we have that the decomposition (4.12) is unique.

Corollary 4.12. Let $X$ be any subset of a pseudo effect algebra with (RDP), and let $F=\{s \in \mathcal{S}(E): X \subseteq \operatorname{Ker}(s)\}$. Every measure $m$ on $E$ can be uniquely decomposed in the form

$$
m=m_{1}+m_{2},
$$

where $m_{1} \in V(F)$ and $m_{2}$ is $V(F)$-singular.

In particular, every state $s$ on $E$ can be uniquely expressed as a convex combination

$$
m=\lambda s_{1}+(1-\lambda) s_{2},
$$

where $s_{1} \in F$ and $s_{2} \in F^{\prime}$. 
Proof. Since $V(F)=\left\{m \in \mathcal{M}^{+}(E): X \subseteq \operatorname{Ker}(m)\right\}$ is by (4.4) $\vee$-closed, the statements follow from Theorem 3.4 .

\section{REFERENCES}

[Alf] E.M. Alfsen, "Compact Convex Sets and Boundary Integrals", Springer-Verlag, Berlin, 1971.

[AlSc] E.M. Alfsen, F.W. Schultz, "State Spaces of Operator Algebras", Birkhäuser, BostonBasel-Berlin, 2001.

[Dvu0] A. Dvurečenskij, "Gleason's Theorem and Its Applications”, Kluwer Academic Publisher, Dordrecht/Boston/London, 1993, 325+xv pp.

[Dvu] A. Dvurečenskij, Central elements and Cantor-Bernstein's theorem for pseudo-effect algebras, J. Austral. Math. Soc. 74 (2003), 121-143.

[Dvu1] A. Dvurečenskij, States on pseudo MV-algebras, Studia Logica 68 (2001), 301-327.

[Dvu2] A. Dvurečenskij, Pseudo MV-algebras are intervals in $\ell$-groups, J. Austral. Math. Soc. 72 (2002), 427-445.

[Dvu3] A. Dvurečenskij, The lattice and simplex structure of states on pseudo effect algebras, submitted. http://arxiv.org/submit/103087

[Dvu4] A. Dvurečenskij, Every state on interval effect algebra is integral, J. Math. Phys. 51 (2010), 083508-12. DOI: 10.1063/1.3467463

[DvPu] A. Dvurečenskij, S. Pulmannová, "New Trends in Quantum Structures", Kluwer Acad. Publ., Dordrecht, Ister Science, Bratislava, 2000.

[DvVe1] A. Dvurečenskij, T. Vetterlein, Pseudoeffect algebras. I. Basic properties, Inter. J. Theor. Phys. 40 (2001), 685-701.

[DvVe2] A. Dvurečenskij, T. Vetterlein, Pseudoeffect algebras. II. Group representation, Inter. J. Theor. Phys. 40 (2001), 703-726.

[DDP] A. Dvurečenskij, P. de Lucia, E. Pap, On a decomposition theorem and its applications, Math. Japonica 44 (1996), 145-164.

[DeMo] P. De Lucia, P. Morales, Decomposition theorems in Riesz spaces, Proc. Amer. Math. Soc. 120 (1994), 193-202.

[FoBe] D.J. Foulis, M.K. Bennett, Effect algebras and unsharp quantum logics, Found. Phys. 24 (1994), 1325-1346.

[GeIo] G. Georgescu, A. Iorgulescu, Pseudo-MV algebras, Multi. Val. Logic 6 (2001), 95-135.

[Goo] K.R. Goodearl, "Partially Ordered Abelian Groups with Interpolation", Math. Surveys and Monographs No. 20, Amer. Math. Soc., Providence, Rhode Island, 1986.

[Hal] P.R. Halmos, "Measure Theory", Springer-Verlag, New York, Heidelberg, Berlin, 1988.

[Rav] K. Ravindran, On a structure theory of effect algebras, PhD thesis, Kansas State Univ., Manhattan, Kansas, 1996.

[Rut1] G.T. Rüttimann, Decomposition of cone of measures, Atti Sem. Mat. Fis. Univ. Modena 38 (1990), 109-121.

[Rut2] G.T. Rüttimann, Weakly purely additive measures, Canad. J. Math. 46 (1994), 872-885.

[YoHe] K. Yosida, E. Hewitt, Finitely additive measures, Trans. Amer. Math. Soc. 72 (1952), 44-66. 